\title{
Diffuse myocardial fibrosis by post-contrast T1- time predicts outcome in heart failure with preserved ejection fraction
}

\author{
Beatrice A Marzluf, Diana Bonderman, Caroline Tufaro, Stefan Pfaffenberger, Alexandra Graf, Martin Hülsmann, \\ Irene M Lang, Richard Pacher, Gerald Maurer, Julia Mascherbauer
}

From 16th Annual SCMR Scientific Sessions

San Francisco, CA, USA. 31 January - 3 February 2013

\section{Background}

Diffuse myocardial fibrosis plays a key role in disease progression of heart failure with preserved ejection fraction (HFPEF). Recently it was shown that diffuse myocardial fibrosis is strongly related to post-contrast longitudinal relaxation (T1) time by cardiac magnetic resonance imaging (CMR). The aim of our study was to assess diffuse myocardial fibrosis by CMR T1-mapping in HFPEF patients and test its predictive value.

\section{Methods}

HFPEF was defined as serum NT-proBNP levels > $220 \mathrm{pg} / \mathrm{ml}$, E/e by echocardiography $\geq 8$, signs or symptoms of heart failure and preserved left ventricular ejection fraction $(\mathrm{EF} \geq 50 \%)$.

63 HFPEF patients and 37 controls were prospectively evaluated. All patients underwent right heart catheterization. CMR studies included the assessment of cardiac function and dimensions by standard cine sequences. Myocardial T1-mapping was performed 15 minutes after a gadolinium bolus using an inversion recovery sequence.

\section{Results}

Post-contrast T1 was significantly correlated with variables reflecting left ventricular filling pressure $(\mathrm{E} / \mathrm{e} \mathrm{p}=0.001, \mathrm{R}=-0.33$; left atrial size, $\mathrm{p}=0.008, \mathrm{R}=-0.27$ ) and pulmonary vascular resistance $(\mathrm{p}=0.004, \mathrm{R}=-0.36)$.

Patients were followed for a median (range) of 12.9 (0.5-23.1) months. By Kaplan-Meier analysis, event-free survival was significantly worse in patients with T1-times below the median of $388.2 \mathrm{~ms}(\log$ rank $\mathrm{p}=0.007)$. By multivariable Cox regression including baseline characteristics, invasive hemodynamics, renal function, and CMR imaging variables, only post-contrast $\mathrm{T} 1$ time $(\mathrm{p}=0.015)$ and left atrium area $(\mathrm{p}=0.029)$ remained independent predictors of event-free survival.

\section{Conclusions}

Our data suggest that post-contrast T1-mapping is a promising tool for the assessment of diffuse myocardial fibrosis. It is closely linked to variables reflecting impaired left ventricular relaxation and hemodynamic variables like pulmonary vascular resistance. Post-contrast T1 time in HFPEF patients outperformed invasive hemodynamics in the multivariable analysis as an independent predictor of event-free survival.

\section{Funding}

none.

Published: 30 January 2013

doi:10.1186/1532-429X-15-S1-M6

Cite this article as: Marzluf et al:: Diffuse myocardial fibrosis by postcontrast T1-time predicts outcome in heart failure with preserved ejection fraction. Journal of Cardiovascular Magnetic Resonance 201315 (Suppl 1):M6.

Cardiology, Medical University of Vienna, Vienna, Austria

(c) 2013 Marzluf et al; licensee BioMed Central Ltd. This is an Open Access article distributed under the terms of the Creative Commons 